\title{
Predicting Radio Frequency Radiation from Mobile Communication Base Stations
}

\author{
A. F. Ismail, N. I. Sidek, K. Abdullah, and W. Hashim
}

\begin{abstract}
While the spectrum growth is expected to increase exponentially in the future years to come, the imminent query would be as whether the current spectrum management process is capable of fulfilling all future requirements. Public interest in the potential health issues relating to cellular or mobile communication base station (BS) and other radio communication facilities emphasize the importance of having an accessible and easy to understand information on electromagnetic (EM) and radio frequency radiation (RFR) levels in the surrounding environment. The information is considered critical in order to assess the potential radiation level prior and during the roll-out of planned or future services such as the 4G, Terrestrial Trunked Radio (TETRA), Worldwide Interoperability for Microwave Access (WiMax), Local Multipoint Distribution Service (LMDS) and others. The paper outlines the attempts of developing a specialized software tool that can gauge the RFR concentration within a particular area. The tool encompasses; among many, the capability of facilitating as well as ensuring that the RFR monitoring activities and planning can be carried out efficiently and effectively.
\end{abstract}

Index Terms-Radio frequency, radiation, base stations, prediction tool.

\section{INTRODUCTION}

Some concerns had been voiced and raised as early as in the 1970s by local communities that magnetic fields radiated from power lines were causing cancer to children [1]. Subsequent epidemiological studies however did not manage to find any conclusive connection between cancer and power lines [2]. Similar anxieties were brought up in the case of wireless communications. Research findings on these issues somehow continue to contradict one another. Moreover, the scientific studies on the possible adverse health effects from low level of RFR emanating from mobile phones and the BS have been found to be inclusive even under long term exposure [3]. The World Health Organization (WHO) even release notifications that "none of the recent reviews have categorically concluded that the exposure of RF fields from mobile phones or their base stations inflicts any adverse health consequences" [4].

Even so, latent harms of RFR should not completely be

Manuscript received February 15, 2013; revised April 12, 2013. The work is currently being funded by the International Islamic University Malaysia's Research University Initiatives RU2011.

A. F. Ismail, N. I. Sidek, and K. Abdullah are with the faculty of Engineering, International Islamic University Malaysia, Kuala Lumpur, Malaysia (e-mail: af_ismail@iium.edu.my, nizzah@iium.edu.my, khaizuran@iium.edu.my).

W. Hashim is with the MIMOS Berhad, Technology Park Malaysia, Kuala Lumpur, Malaysia (e-mail: wahidah.hashim@mimos.my). taken for granted. In principle, radiation can harm human tissues if humans are exposed to high levels of RFR over an extended period of time. RFR has the capacity to heat up human tissue. Tissue damage due to RFR exposure occurs as the human body is not capable of dissipating excessive amounts of heat [5]. In addition, there are also added concerns associated to the non-ionizing radiation effects such as sleeping disorders, epidemiological, neuropsychiatric and psychological consequences; where RFR might not portray to be capable of inflicting any long-term effects. These effects may not cause any direct damage to tissue, yet scientists are still unsure about whether prolonged RFR exposure could create health problems [6].

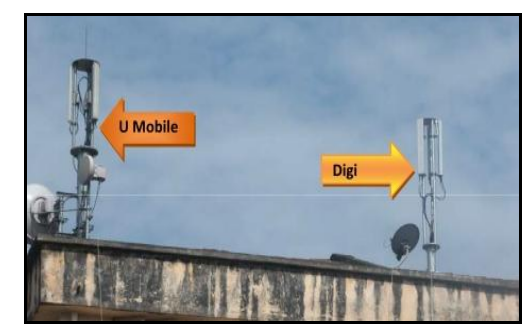

Fig. 1. Typical base station installations of two national service providers on a building rooftop

There have been numerous guidelines and regulations on the radiation protection such as from the International Committee of Non-Ionizing Radiation Protection (ICNIRP) and National Radiological Protection Board (NRPB). However, there have been disagreements on the safety margin level proposed by ICNIRP back in 1999. As a result, the various governing authorities had concluded to make the permissible RFR levels much lower than what ICNIRP had recommended as they believe that in this exposure level might have eventually some thermal effects on human body [7], [8].

Fig. 1 shows typical BS installations by two Malaysian mobile operators that can be easily noticeable on many building rooftops. With the influx sudden increase of BS installations, the obvious challenge now is not only limited to the impending review of permissible RFR applicable standard level, to the present situation and time, but also on the method of determining the RF exposure and right tool or tools capable of accurately predict and measure the RFR levels.

\section{Algoritm AND Modelling}

\section{A. Associated Algorithm}

Whilst coordinating and managing the spectrum, relevant 
authorities are expected to employ RFR prediction tool. The said tool's database is expected to include the service area map which contains not only data on BS locations and their particular frequency allocations, but also their projected radiation levels. Physical RFR measurement may well continually serve as the most accurate method to ascertain the RFR level at specific locations; however a prediction tool which is competent to estimate RFR levels with high degree of accuracy can rather be very useful and practical. It will certainly facilitate the pre-processing measurement steps, especially when there are countless numbers of BS sites to be assessed all at the same time. At present the current research undertakings are very much concentrated on developing projected tool, capable of answering the forthcoming requirements in RFR monitoring.

In general, the safety distance for each individual transmitting antenna within its frequency band can be estimated using the following parameters: a) Antenna technical parameters i.e. frequency, effective isotropic radiated power (EIRP), power, gain, dimension, and etc. b) Antenna deployment details i.e. antenna height, position, etc. Fig. 2 illustrates a depiction of the point of interest (POI) and range of interest (ROI) from the antenna in the side view.

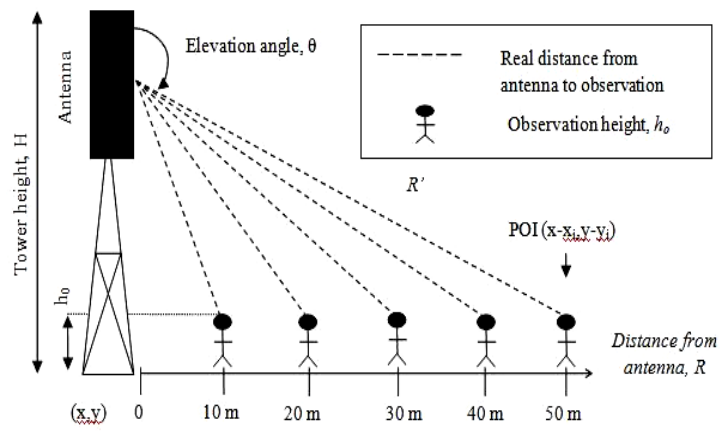

Fig. 2. Illustration of RFR estimation from antenna in vertical view for single BS assessment [9]

With acknowledged observation height, $\mathrm{h}_{\mathrm{o}}$ and the tower height, $\mathrm{H}$ is given; the real distance, $\mathrm{R}$ ' can be determined by

$$
R^{\prime}=\left(H-h_{0}\right)^{2}+R^{2}
$$

and by using Pythagorean Theorem the real distance, $\mathrm{R}^{\prime}$ is established by

$$
R^{\prime 2}=\left(H-h_{0}\right)^{2}+\left(x-x_{i}\right)^{2}+\left(y-y_{i}\right)^{2}
$$

where $\mathrm{i} \in\{1,2, . ., \mathrm{n}\}$. The elevation angle, $\theta$ in this case is formulated by the equation

$$
\theta=\pi-\tan ^{-1}\left(\frac{H-h_{0}}{x}\right)
$$

The power density in $\mathrm{W} / \mathrm{m}^{2}$ for $\mathrm{S}$ equation due to a point in a free space far field (FF) region in relation to (1) is specified as

$$
S=\frac{P G(\theta, \varphi)}{4 \pi R^{\prime 2}}=\frac{P G(\theta, \varphi)}{4 \pi\left[\left(H-h_{0}\right)^{2}+\left(x-x_{i}\right)^{2}+\left(y-y_{i}\right)^{2}\right]}
$$

The same applies to the computation of power density in the near field (NF) region where it can be articulated as

$$
S=\sum_{i=1}^{n} S_{i}=\sum_{i=1}^{n} \frac{P G(\theta, \varphi)}{4 \pi\left[\left(H-h_{0}\right)^{2}+\left(x-x_{i}\right)^{2}+\left(y-y_{i}\right)^{2}\right]}
$$

where $\theta_{1 / 2}$ is the horizontal half power bandwidth (HPBW). In the case of multiple antennas, the overall power density due to all antennas in the FF free space can be put together as:

$$
S=\sum_{i=1}^{n} S_{i}=\sum_{i=1}^{n} \frac{P G(\theta, \varphi)}{4 \pi\left[\left(H-h_{0}\right)^{2}+\left(x-x_{i}\right)^{2}+\left(y-y_{i}\right)^{2}\right]}
$$

where the $i^{\text {th }}$ one may be embodied by its power $P_{i}$, gain relative to the isotropic antenna in a given direction, $G_{i}$ and coordinates relative to a certain origin $\left(x_{i}, y_{i}\right)$ where $i €$ $\{1,2, \ldots, n\}$.

Fig. 3 portrays the diagram involving the modeling of multiple transmitters at a specific point of interest within a co-located site. The contribution of each antenna on a POI is computed and summed to the rule in (6). For each operating frequency, the overall exposure at the POI is the vector sum of each power density or electric field level from each antenna. In the illustrated example, the total power density exposure, $S$ at POI is $\sqrt{ }\left(S_{R 1}{ }^{2}+S_{R 2}{ }^{2}\right)$ and the same concept applies when calculating the E-field exposure. The association between the power density and E-field is given by $E=\sqrt{ } \mathrm{S} \times 120 \pi$. Fig. 4 presents the associated flow chart for performing the assessment of RF exposure for a BS site with multiple transmitters. For instances when assessment on multiple antennas within a co-located site reveal level that exceeds the safety limits, the highest contributor of the RFR levels must be adjusted and/or re-planned.

\section{B. Multiple Transmitters at Co-Located Site Modelling}

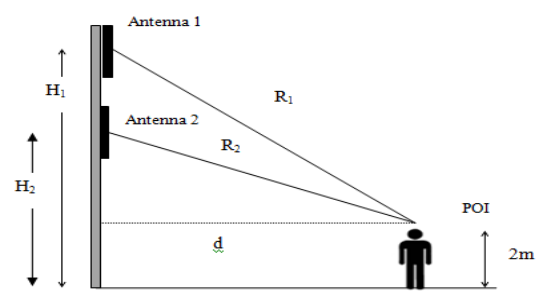

Fig. 3. Co-located transmitters at single site with reference to ground level exposure assessment (of $\sim 2$ meters height)

\section{Multiple Transmitters at Non Co-Located Site(s) Modelling}

Several ideas relating to applicable mitigation techniques had been mooted and are now currently being pursed and devised. The proposals encompasses mitigation techniques that entail the decrease of power whilst using high gain antennas, increasing the antenna height from round as well as adjusting the tilting angle of the antenna [10]. These circumstances are illustrated by Fig. 5 and Fig. 6. In Fig. 5, The RFR contribution on POI is the cumulative of all transmitters on the multiple towers involved. In the case of Fig. 6, the assessments are done as in case in Fig. 5 except that the distance, $d$ of the antennas toward the POI might be different depending on the position of the antenna. For case as in Fig. 6, the total exposure is calculated on different POIs, hence the safety distance varies according to the antenna height from ground of the POI and the orientation of the 
antenna. The assessment of the cases depicted in both figures can be evaluated following the highlighted process as shown in the flow chart labeled as Fig. 4.

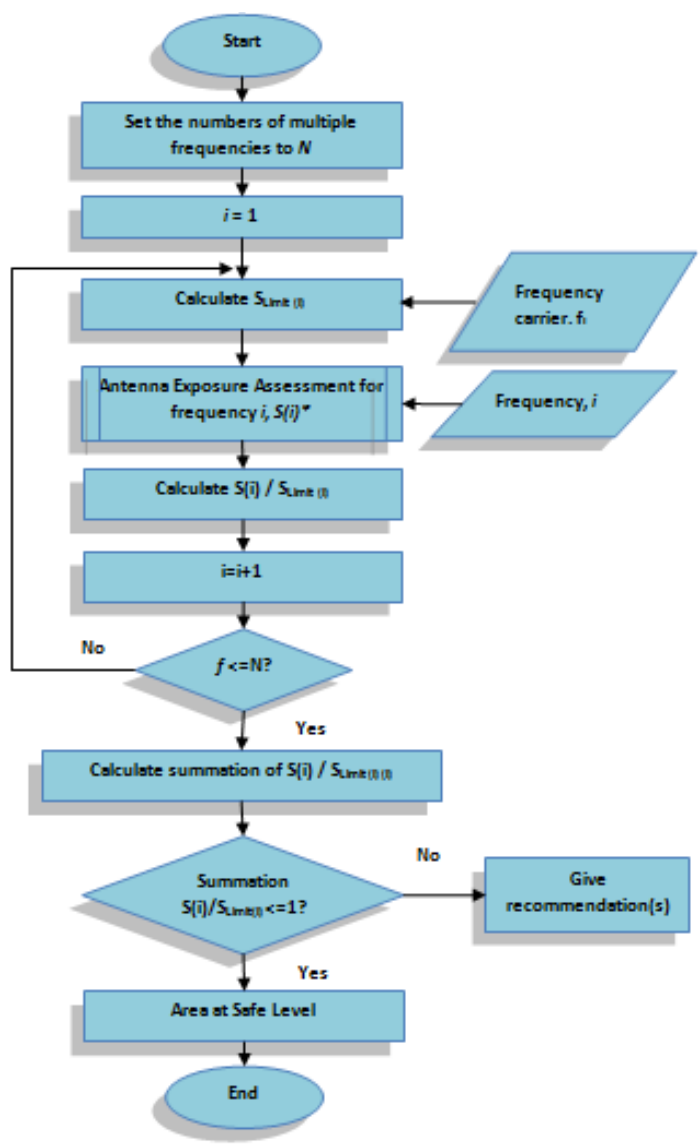

Fig. 4. Flow chart of RF exposure assessment module for a multiple transmitters at a co-located site (share tower/mast) or multiple transmitters at non co-located sites

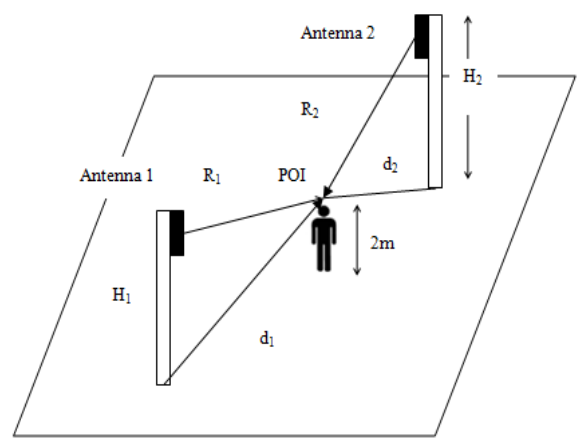

Fig. 5. Transmitters at non co-located sites contributing to an RF field POI

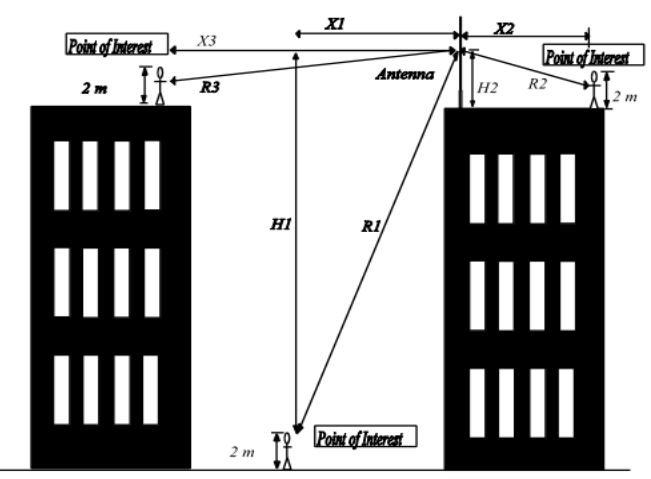

Fig. 6. RFR distribution from a single roof top antenna to various locations

\section{SOFTWARE OVERVIEW AND DESIGN}

This paper intends to underline the development of a specialize software tool incorporating procedures capable of facilitating as well as ensuring that the RFR monitoring activities and planning can be done efficiently and effectively. The chosen models and algorithm are further implemented using a Geographic Information System (GIS) software called SAGA version 2.0.4 with $\mathrm{C}++$ environment. GIS is in principle a computer-based technology for collecting, managing, analyzing, modeling and presenting geographic data for wide range of applications. Among it common usages are such as for remote sensing, planning, surveying, radio wave propagation planning and others including RFR and EM field monitoring. Exploited GIS can be devised to offer integration of the RFR prediction from BS, geographical information and database, in the hope to ensure the RFR level is within the permissible values at all times. Data can be gathered, stored and managed in an efficient way and the process of administering as well as keeping track of the RFR data replaces the physical map storage structure.
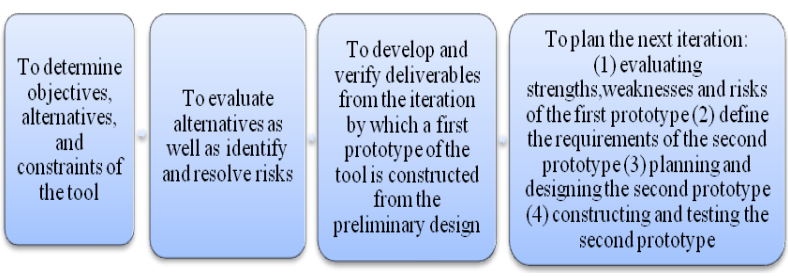

Fig. 7. Four basic quadrants of spiral model adopted

In order to design a tool or software, a specific software development model must be followed. The common model applied is the spiral model which is a software development process combining elements of both design and prototyping stages [11]. Each trip around the spiral traverses four basic quadrants as featured in Fig. 7. The development of this tool can be considered as the first prototype, where large room of improvements can be done in the future. The overall structure of the proposed tool is as depicted in Fig. 8.
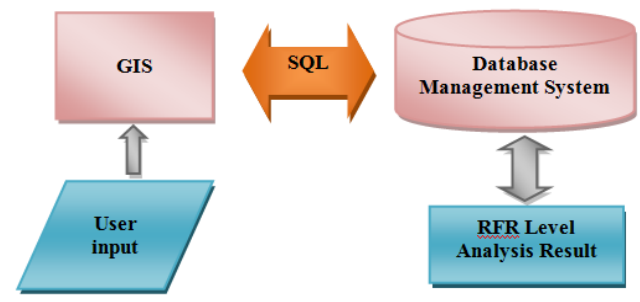

Fig. 8. The developed RFR Tool's overall structure [9]

\section{PRELIMINARY FINDINGS}

An initial module called 'Antenna Radiation' was created as shown in Fig. 9. Under this module, a function called 'Map Existing Antenna' module that estimates the RFR from existing BSs that are already present in the real environment was built. Zoom out view functionality was developed for the estimated area for multiple BS (assuming Omni-directional pattern) emulating previous investigators' approach [12], [13]. The centers of both antennas are quite near and radiation pattern expected to overlap each other. There are parameters 
that need to be entered into the system to enable the determination of RFR levels and these data will be automatically kept into the database. The calculation module trails the flow chart shown in Fig. 4, but with the advantages of radiation pattern information.

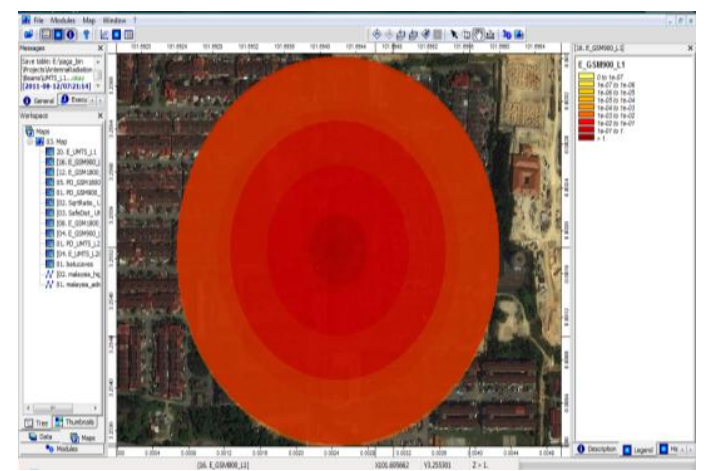

Fig. 9. Antenna radiation from a single BS site with co-located multiple transmitters

Upon executing the calculation using the 'Map Existing Antenna' module, results then will be displayed according to the power density values of each cell, the E-field value, squared ratio of the power density over reference level and the safety distance of each antenna. The outputs of the simulated RFR from the antennas can be displayed in a 2-D graphical visualization on top of the base map. In the case of overlapping radiation patterns for multiple antennas, it is found out that the overlapping regions are misaligned between each other, affecting the values of the overlapping cells as illustrated by Fig. 10. This therefore requires complex computations to determine the resultant power density (PD) value on each centre of a cell. For the time being, calculations are based on limited data, thus producing only a general view on exposure levels within the investigated sites.

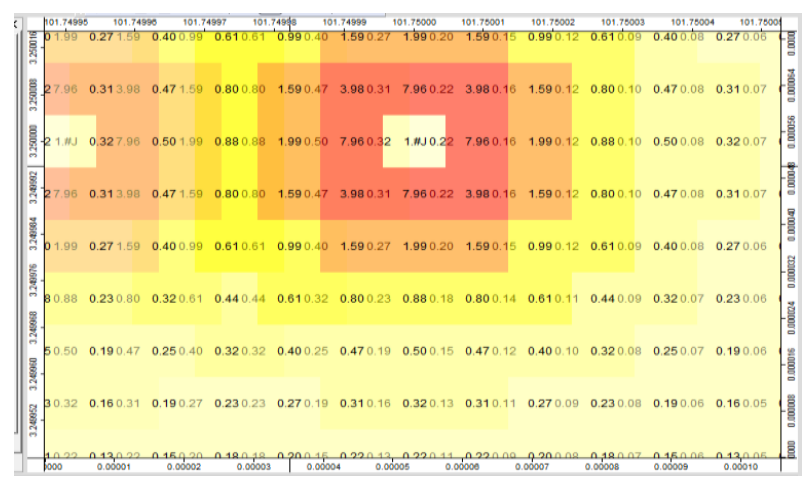

Fig. 10. Limitations in the case of overlapping region for multiple antennas

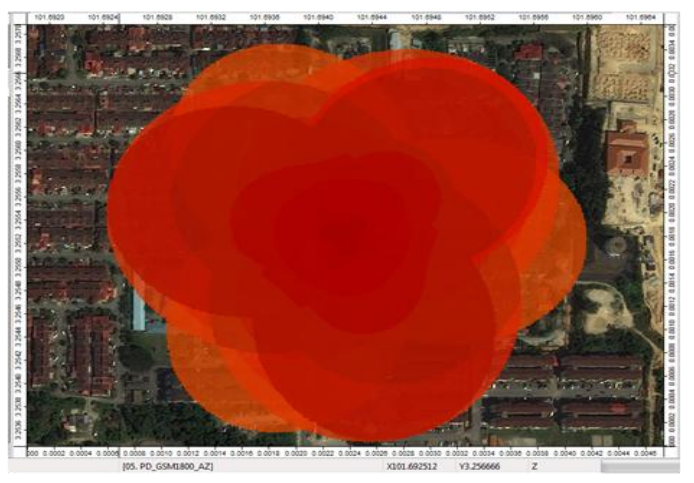

Fig. 11. Multiple BS and their RFR assessment
Solutions to overcome the mentioned limitations had been identified and developed. Fig. 11 shows the snapshot of the affected areas from both BSs using the proposed tool. Since both BSs are located quite near from each other, each radiation overlaps each other and the antenna has an Omni-directional pattern due to the limitations of antenna radiation pattern. Due to that, early finding suggests that the RFR level will be overly predicted.

\section{CONCLUSION}

Any tool is predictive and cannot absolutely define the real environment. However, prediction tools modeling have two distinct major advantages over on-site measurements. First, it can simulate the parameters for each antenna whether or not the transmitter is actually fired up. Second, modeling does not involve excessive field time. In the proposed tool, one need only know the location of the antennas and their frequencies, powers, and gains to provide adequate input to the modeling tool. Additional data such as the radiation pattern, orientation, height, loss etc however adds to the accuracy of the estimation. Measurements must be made over time and then mapped. The proposed RFR prediction tool can certainly be very helpful in determining field levels based on the prediction models. The results however for now at best, are only as valid as the input data.

\section{ACKNOWLEDGMENT}

The authors acknowledge the Research Management Centre of the International Islamic University Malaysia (IIUM) for the financial support and would like to express special appreciation to the Malaysian Communication and Multimedia Commission for the technical guidance and assistance. The reported research findings are part of the deliverables for the IIUM's funded studies

\section{REFERENCES}

[1] N. Wertheimer and E. Leeper, "Electrical wiring configurations and childhood cancer," American Journal of Epidemiology, vol. 109, no. 3 , pp. 273-284, Aug. 1979.

[2] R. A. Kleinerman, W. T. Kaune, E. E. Hatch, S. Wacholder, M. S. Linet, L. L. Robison, S. Niwa, and R. E. Tarone, "Are children living near high voltage power lines at increased risk of acute lymphocytic leukemia?” American Journal of Epidemiology, vol. 151, no. 5, pp. 512-515, May 2000.

[3] J. C. Lin, "Personal telecommunication radiation safety and the precautionary principle," IEEE Antennas and Propagation Magazine, vol. 45, no. 2, pp. 142-145, April 2003.

[4] Electromagnetic fields and public health: Mobile telephone and their base stations. (2007). [Online]. Available: http://www.who.int/mediacentre/factsheets/fs 193/en/

[5] A. D. Usman and W. F. W. Ahmad, "RF EM exposure: the issues, the debate and the realities," in Proc. the IEEE International RF and Microwave Conference, 2008, pp. 41-45.

[6] Scientific Committee on Emerging and Newly Identified Health Risks, "Preliminary opinion on possible effects of electromagnetic fields on human health," in Brussels: European Commission Report, 2007.

[7] "Guidelines for Limiting Exposure to Time-Varying Electric and Magnetic Fields (1 Hz - $100 \mathrm{kHz}$ )," in Health Physics, 2010, vol. 99, no. 6, pp. 818-836.

[8] Information and Communication Technologies Authority (ICTA), "Regulation on determination, measurement methods and supervision of the limit values of the electromagnetic field force from the fixed telecommunication devices operating within the frequency range of 10 kHz-60 GHz," in Turkey's Official Gazette, no. 24460, December 7 , 2001 . 
[9] A. F. Ismail, H. A. Mohd Ramli, N. I. Sidek, and W. Hashim, "Development of radio frequency radiation (RFR) prediction tool," in Proc. 18 $18^{\text {th }}$ Asia-Pacific Conference on Communication, $2012 \mathrm{pp}$. 204-207.

[10] International Telecommunication Union, "Mitigation techniques to limit human exposure to EMFs in the vicinity of radiocommunication stations," in ITU-T Recommendation K.70, Geneva, 2007.

[11] B. Boehm, A. Egyed, J. Kwan, D. Port, A. Shah, and R. Madachy, "Using the win win spiral model: A case study," Journal of Computer Engineering, vol. 31, no. 7, pp. 33-44, July 1998.

[12] S. C. D. Cruz, M. H. C. Dias, and G. L. Siqueira, "Selective RF power density measurements radiated by mobile telephony RBS in Rio de Janeiro city," European Microwave Conference, vol. 3, pp. 48-51, 2005.

[13] J. S. Seybold, Introduction to RF propagation, New Jersey, John Wiley \& Sons, Inc., 2005, ch. 2.

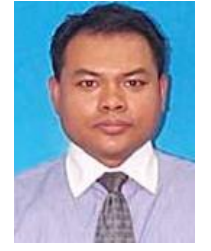

A. F. Ismail is a lecturer at the Faculty of Engineering, International Islamic University Malaysia. He completed his B. Electrical Eng. at Gannon University, Pennsylvania, USA with Cum Laude Latin honors in 1994. He holds an MSc from Essex University, UK and $\mathrm{PhD}$ from of Bath University, UK. His research interests include millimeter and microwave propagation studies and Cognitive Radio applications. He is a registered BEM's Professional Engineer and also a Senior Member of the IEEE.

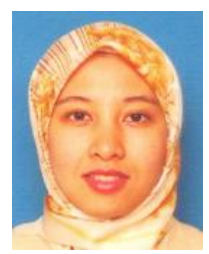

W. Hashim received his bachelor degree in Information Technology, Business Management and Language from University of York, UK in 1999. She then pursued her MSc in Multimedia Technology at University of Bath, UK in 2001. She completed her PhD studies from King's College London, UK in 2008 in the field of Telecommunication Engineering. She is currently a staff researcher at the Wireless Communication Cluster, MIMOS Berhad with focus in cognitive radio, WLAN, OFDM, space-time coding, MIMO systems and wireless system. 